TITLE:

\title{
Magnetization switching of a magnetic wire with trilayer structure using giant magnetoresistance effect
}

\section{$\operatorname{AUTHOR}(\mathrm{S}):$}

Shigeto, K; Okuno, T; Shinjo, T; Suzuki, Y; Ono, T

\section{CITATION:}

Shigeto, K ... [et al]. Magnetization switching of a magnetic wire with trilayer structure using giant magnetoresistance effect. JOURNAL OF APPLIED PHYSICS 2000, 88(11): 66366644

ISSUE DATE:

2000-12-01

URL:

http://hdl.handle.net/2433/50347

\section{RIGHT:}

Copyright 2000 American Institute of Physics. This article may be downloaded for personal use only. Any other use requires prior permission of the author and the American Institute of Physics. 


\title{
Magnetization switching of a magnetic wire with trilayer structure using giant magnetoresistance effect
}

\author{
K. Shigeto, ${ }^{\text {a) }}$ T. Okuno, and T. Shinjo \\ Institute for Chemical Research, Kyoto University, Uji 611-0011, Japan \\ Y. Suzuki \\ Electrotechnical Laboratory, Material Science Division, Umezono, Tsukuba 305-8568, Japan \\ T. Ono \\ Faculty of Science and Technology, Keio University, Yokohama 223-8522, Japan
}

(Received 5 June 2000; accepted for publication 13 September 2000)

\begin{abstract}
The switching fields of magnetic wires with trilayer structure consisting of $\mathrm{NiFe} / \mathrm{Cu} / \mathrm{Co}$ were investigated using giant magnetic resistance effect. The switching fields of both magnetic layers were observed to be inversely proportional to wire width $(150-520 \mathrm{~nm})$. We found that the magnetization of the NiFe layer switches under much lower applied field than in the case of single layer structure by the assistance of the stray field from the magnetic charge of Co at the edge of the wire. Attaching a pad at one end of the wire causes drastic decrease of the switching field. We investigated pad shape dependence of the switching field of the Co layer. For the sample with a square pad we measured the temperature dependence of the switching field between 5 and $300 \mathrm{~K}$. The dependence at low temperatures between 5 and $50 \mathrm{~K}$ can be described by the model on thermally assisted magnetization reversal over a simple potential barrier. (c) 2000 American
\end{abstract} Institute of Physics. [S0021-8979(00)03924-4]

\section{INTRODUCTION}

Magnetic behaviors of mesoscopic systems have become an updated topic both from scientific and technological points of view. However, in general, experimental studies of magnetic nanostructures have been made on samples consisting of a large number of nearly identical particles, since conventional measurement techniques are not sensitive enough to measure the magnetization of a single particle. Most of single-particle or single-wire properties are inevitably hidden behind the distribution of size or shape. Recently experimental studies of an individual magnetic particle have become possible with the techniques such as magnetic force microscopy (MFM), ${ }^{1}$ Lorentz microscopy, ${ }^{2}$ electron holography, ${ }^{3}$ ballistic Hall micromagnetometry, ${ }^{4}$ and microsuperconducting quantum interference device (SQUID) magnetometry. ${ }^{5,6}$ However, these experiments are only carried out under limited temperatures and magnetic fields.

As reported in a previous paper, ${ }^{7}$ we demonstrated that a very small magnetization change in a single $\mathrm{NiFe}(20 \mathrm{~nm}) /$ $\mathrm{Cu}(10 \mathrm{~nm}) / \mathrm{NiFe}(5 \mathrm{~nm})$ trilayer wire with $0.5 \mu \mathrm{m}$ width can be detected by using the noncoupled type giant magnetoresistance (GMR) effect. In very narrow ferromagnetic wires, the magnetization is restricted to direct parallel to the wire axis due to the magnetic shape anisotropy. Normally, it is considered that magnetization reversal takes place by nucleation and propagation of a magnetic domain wall. ${ }^{8}$ In this case the GMR change is directly proportional to the magnitude of the switching layer magnetization. Therefore, we

\footnotetext{
a) Author to whom correspondence should be addressed; electronic mail: shigeto@scl.kyoto-u.ac.jp
}

have already succeeded in measuring the velocity of a magnetic domain wall propagating in a wire. ${ }^{9}$ It is also an advantage that our detection technique using GMR effect has no limitation on temperature and magnetic field as far as resistance measurement is possible. In this article, we report on the switching field $H_{\mathrm{sw}}$ of the two types of samples consisting of $\mathrm{NiFe} / \mathrm{Cu} / \mathrm{Co}$. One of the two types of wires has a symmetric shape with flat ends, while the other is connected to a pad (large area) at an end (see Fig. 1). We introduced the wire with a pad for the purpose of specifying the direction of domain wall propagation in the magnetization reversal process. ${ }^{10}$ For the wires without a pad, we present the results of width dependence (150-520 nm) and influence on the switching field of the NiFe layer from stray field from the magnetic charge of $\mathrm{Co}$ at the edge of the wire. For the wires (150 nm width) with a pad we present the results of padshape dependence on the switching field of Co layer at $300 \mathrm{~K}$ and temperature dependence over a wide range from 5 to 300 $\mathrm{K}$ specially for the wire with a square pad.

\section{EXPERIMENTAL PROCEDURE}

Figure 1 shows schematic illustrations of the top views of typical wire samples (a) without a pad and (b) with a pad. The wire without a pad has a symmetrical shape with two flat ends, while the wire with a pad is connected to a square pad $\left(0.5 \times 0.5 \mu \mathrm{m}^{2}\right)$. All samples measured in this work have four current-voltage probes made of a nonmagnetic material, $\mathrm{Cu}$, to eliminate the disturbance of magnetization of the wire by magnetic probes. The samples were fabricated onto thermally oxidized Si substrates by means of e-beam lithography and liftoff techniques in two steps as follows. In the first step, a wire was patterned and the trilayer film of NiFe/ 


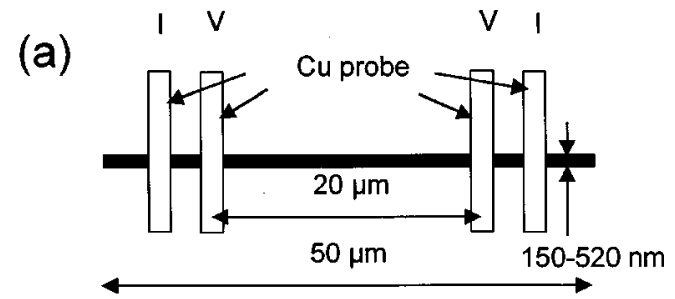

(b)

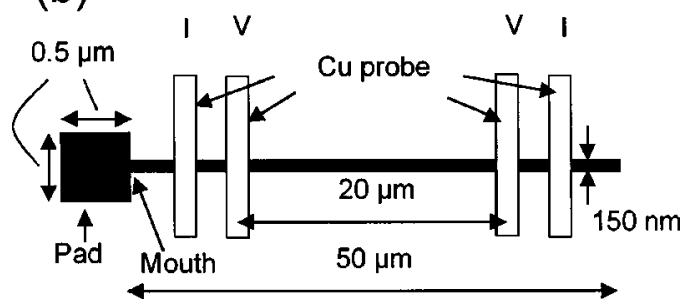

FIG. 1. Schematic illustrations of the top views of the typical two types of wires. Both of them have four current-voltage probes made of a nonmagnetic material, $\mathrm{Cu}$.

$\mathrm{Cu} / \mathrm{Co}$ was deposited at a rate of $0.03 \mathrm{~nm} / \mathrm{s}$ by electron-beam evaporation in a vacuum of $1 \times 10^{-8}$ Torr. In the second step, the $\mathrm{Cu}$ probes were patterned to make electrical contact to the wire. $\mathrm{Cu}$ probes were also deposited by e-gun evaporation at a rate of $0.1 \mathrm{~nm} / \mathrm{s}$. Thickness of the Cu probes is $150 \mathrm{~nm}$. The distance between voltage probes is $20 \mu \mathrm{m}$. All magnetoresistance (MR) measurements were performed with a constant dc current of $5 \mu \mathrm{A}$. Shape and size of the samples were checked by using a scanning electron microscope (SEM) after MR measurements.

\section{RESULTS AND DISCUSSION}

\section{A. Width dependence of the wire without a pad}

Figure 2 shows typical resistance changes of the trilayer systems as a function of applied magnetic field parallel to the wire axis at $300 \mathrm{~K}$; the width of the wires ( $w$ $=150,340,520 \mathrm{~nm}$ ) is marked in each MR curve. The sample shape is also schematically shown in Fig. 2. All wires have the same trilayer structure of $\mathrm{NiFe}(20 \mathrm{~nm}) / \mathrm{Cu}(20 \mathrm{~nm}) / \mathrm{Co}$ $(20 \mathrm{~nm})$. Prior to the measurement, a magnetic field of 3000 Oe was applied in order to align the magnetizations in one direction. Then the resistance was measured with sweeping the field towards the counterdirection at the rate of $6 \mathrm{Oe} / \mathrm{s}$. As far as the counterfield is smaller than the switching field $H_{\text {sw }}$ of the $\mathrm{NiFe}$ layer, both magnetizations of the NiFe and the Co layers align in parallel and the resistance takes the smallest value. When the applied magnetic field exceeds the $H_{\text {sw }}$ of the NiFe layer, the resistance abruptly increases and stays at the largest value until the field reaches the $H_{\text {sw }}$ of the Co layer, and then the resistance abruptly decreases to the smallest value. In this case, the resistance increase at lower field corresponds to the magnetization switching of the $\mathrm{NiFe}$ layer and the resistance decrease at higher field corresponds to the magnetization switching of the Co layer. The sudden leaps of resistance indicate that the magnetic domain wall nucleation field for both $\mathrm{NiFe}$ and Co layers are much larger than the critical field for the wall propagation. The resistivity
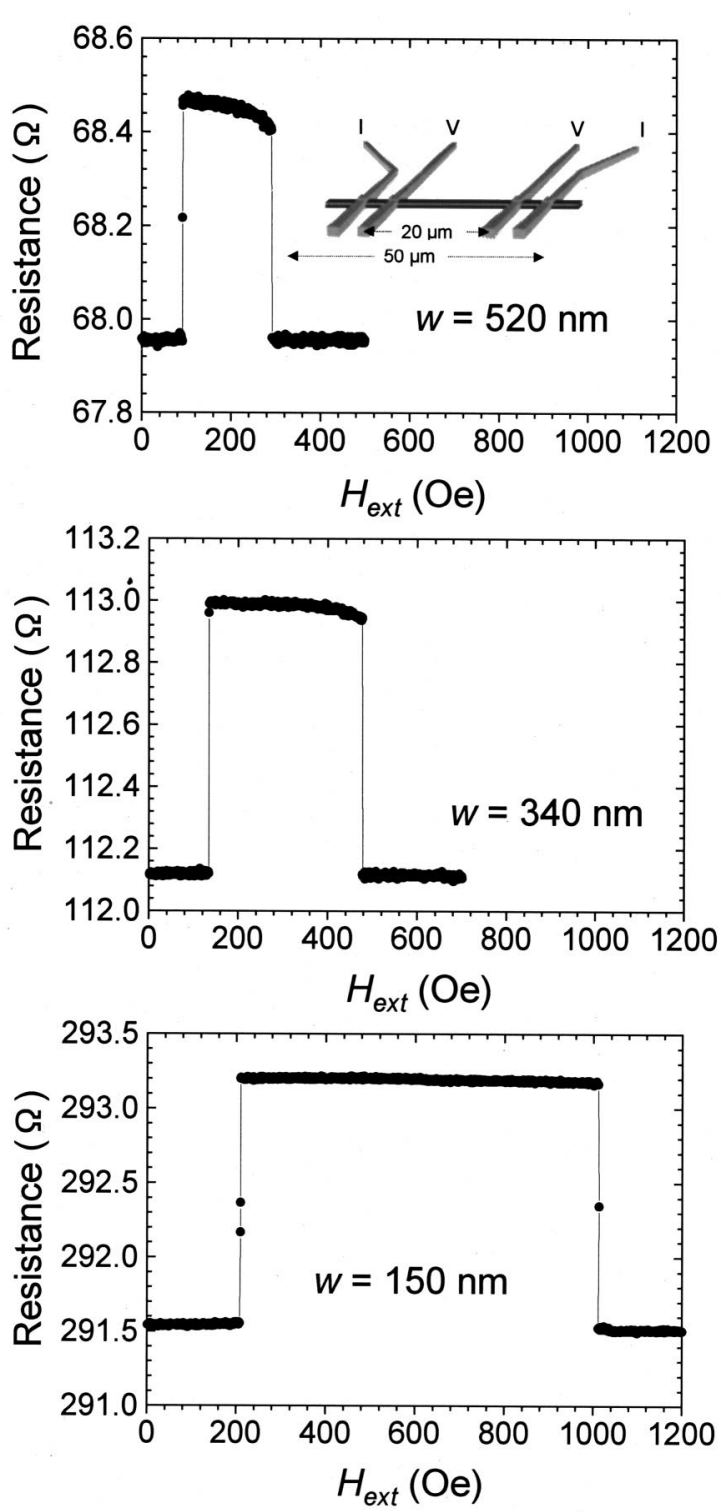

FIG. 2. Resistance as a function of the external magnetic field $H_{\text {ext }}$ parallel to the wire axis at $300 \mathrm{~K}$ for various wire widths 520,340 , and $150 \mathrm{~nm}$. The schematic illustration shows the sample consisting of NiFe $(20 \mathrm{~nm}) / \mathrm{Cu}(20$ $\mathrm{nm}) / \mathrm{Co}(20 \mathrm{~nm})$ for width dependent measurements. Sweep rate of the external field is $6 \mathrm{Oe} / \mathrm{s}$.

at zero field when the magnetization configuration of both magnetic layers parallel is $10.4 \mu \Omega \mathrm{cm}$ for all wires.

The measured $H_{\mathrm{sw}}$ has a stochastic nature. Therefore, we construct histograms of the $H_{\mathrm{sw}}$ 's from 30 MR curves. As shown in Fig. 3, the width of the histograms is narrow enough to discuss the values of the $H_{\text {sw }}$ 's for all cases. In particular, for the Co layer we notice a tendency that the distribution width decreases with decreasing $w$. This is most likely caused by the decreasing of the degree of freedom in the wall nucleation process with decreasing $w$.

We plotted the mean value of the $H_{\mathrm{sw}}$ both for the NiFe and Co layers as a function of $1 / w$ in Fig. 4(a). The $w$ of the wires was determined by SEM. We found that the switching fields of both magnetic layers are proportional to $1 / w$ ( $w$ $=150-520 \mathrm{~nm}$ ). This relation reminds us of an equation of 

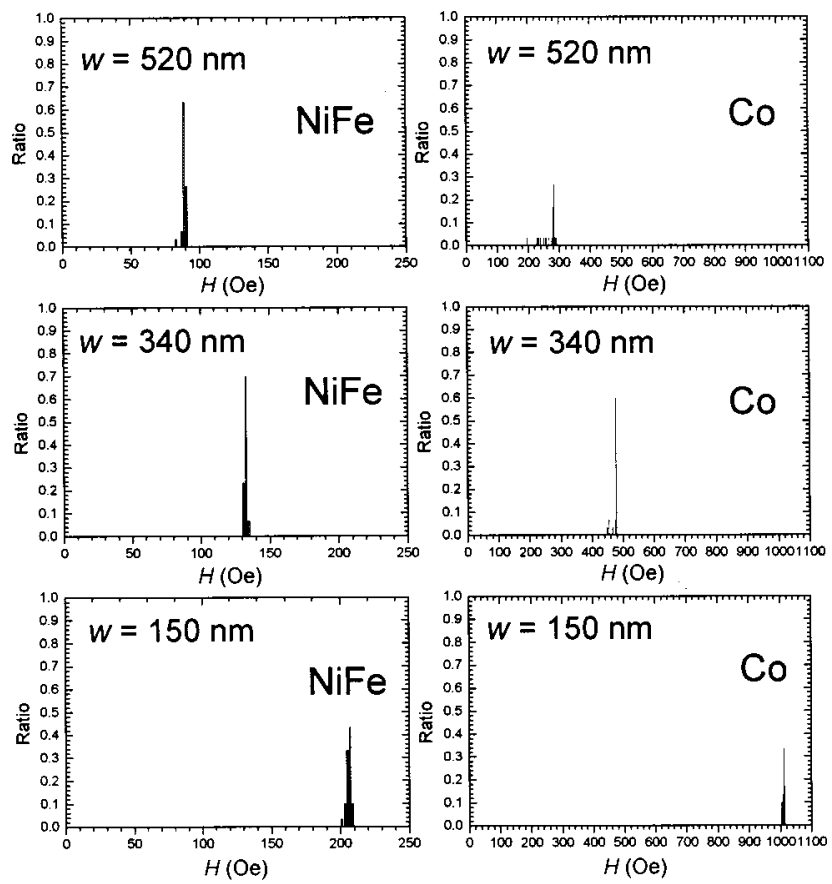

FIG. 3. Histograms of the switching field $H_{\text {sw }}$ of the $\mathrm{NiFe}(20 \mathrm{~nm})$ layer and $\mathrm{Co}(20 \mathrm{~nm})$ layer for various wire widths 520,340 , and $150 \mathrm{~nm}$, obtained by repeating the MR measurements 30 times.

the average hard axis demagnetizing field $H_{d},{ }^{11-14}$ which can be estimated as

$$
H_{d}=\left(\frac{t}{w}\right) \frac{M_{s}}{\mu_{0}}
$$

where $t$ is the film thickness, $M_{s}$ the saturation magnetization, and $\mu_{0}$ the vacuum permeability. Magnetization reversal certainly takes place by nucleation and propagation of the magnetic domain wall because the observed $H_{\text {sw }}$ is much smaller than the $H_{d}$. However the process of the domain wall nucleation was accompanied by the tilt of the magnetization to the hard axis in the plane. Therefore it seems that there is correlation between $H_{d}$ and $H_{\mathrm{sw}}$. In Eq. (1) $H_{d}$ is not only proportional to $1 / w$ but also proportional to $M_{s}$. The ratio of the slopes of the two lines in Fig. 4(a) should then be coincident with the value of $M_{s}^{\mathrm{Co}} / M_{s}^{\mathrm{NiFe}}=1.79 \mathrm{~T} / 1.08 \mathrm{~T}$ $=1.6$, where $M_{s}^{\mathrm{Co}}$ and $M_{s}^{\mathrm{NiFe}}$ are the saturation magnetization

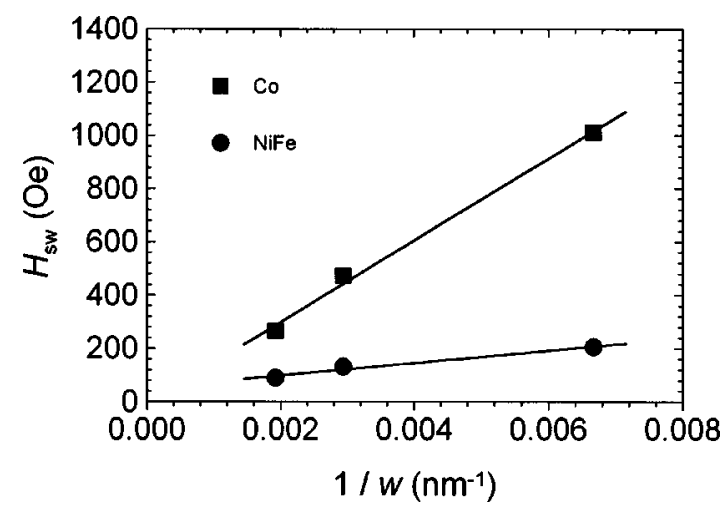

FIG. 4. Average $H_{\mathrm{sw}}$ of the $\mathrm{NiFe}(20 \mathrm{~nm})$ layer and $\mathrm{Co}(20 \mathrm{~nm})$ layer as a function of the inverse of the wire width at $300 \mathrm{~K}$.

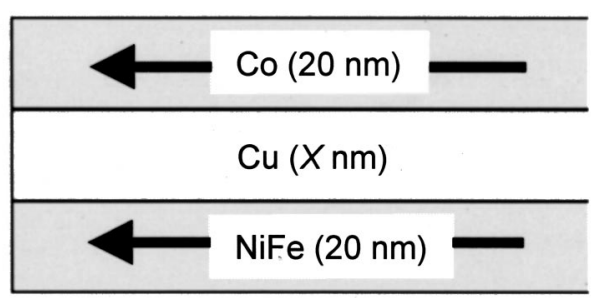

Shigeto et al.
FIG. 5. Schematic illustrations of the side view at the left edge of the trilayer wire. $X$ is $\mathrm{Cu}$ layer thickness and $H(\mathrm{Co})_{\|}$is the parallel component of the stray field $H$ from the magnetic charge of Co layer at the edge of the wire.

of $\mathrm{Co}$ and $\mathrm{NiFe}$, respectively. However the ratio of the two slopes is about 6.6 which is much larger than the estimation. We found this disagreement is caused by the fact that the magnetization of the NiFe layer in the trilayer structure of $\mathrm{NiFe} / \mathrm{Cu} / \mathrm{Co}$ switches under much lower applied field than in the case of single layer structure by the assistance of stray field from the magnetic charge of Co at the edge of the wire. In the next section we discuss this point.

\section{B. Influence of the trilayer structure}

Our samples have trilayer structures to detect the magnetization switching using GMR effect. The magnetization of a magnetic wire is restricted to direct parallel to the wire axis due to the magnetic shape anisotropy. Therefore magnetic charges are located only at both ends of the wire, from which the stray field radially flows out. Of course the magnitude of the stray field rapidly decreases out of the edge and the magnetic field is effective only near the edge. Schematic illustrations of the side view at the left edge in our sample are shown in Fig. 5, where both magnetizations direct parallel. The important thing is that the domain wall nucleation which is closely related with $H_{\mathrm{sw}}$ takes place at the edge of the wire. Therefore there is a possibility that the $H_{\mathrm{sw}}$ 's observed in this study are values affected by the stray field from the magnetic charge of the another magnetic layer.

Only for the layer with the smaller $H_{\mathrm{sw}}$, the NiFe layer in this article, we can detect the effect of the stray field from the such MR measurements as shown in Fig. 6. The MR curve in Fig. 6 is for the wire $(w=150 \mathrm{~nm})$ with a trilayer structure of $\mathrm{NiFe}(20 \mathrm{~nm}) / \mathrm{Cu}(20 \mathrm{~nm}) / \mathrm{Co}(20 \mathrm{~nm})$. After the magnetic field was swept from -3000 to $400 \mathrm{Oe}$ at $6 \mathrm{Oe} / \mathrm{s}$ parallel to the wire axis, the field was swept to the counterdirection at the same rate. We define the $H_{\mathrm{sw}}$ with sudden 

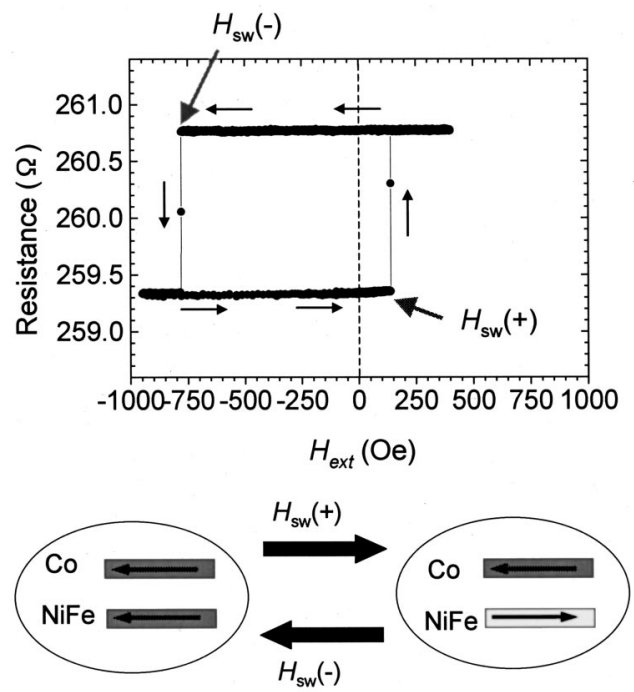

FIG. 6. Resistance as a function of the external magnetic field $H_{\text {ext }}$ parallel to the wire axis at $300 \mathrm{~K}$ for $150 \mathrm{~nm}$ wire width. How the external field was applied is shown by arrows. The measured wire consists of $\mathrm{NiFe}(20 \mathrm{~nm}) /$ $\mathrm{Cu}(20 \mathrm{~nm}) / \mathrm{Co}(20 \mathrm{~nm})$. The magnetization alignment before and after the two $H_{\text {sw }}$ 's are schematically shown.

resistance increase as $H_{\mathrm{sw}}(+)$ and the $H_{\mathrm{sw}}$ with sudden resistance decrease as $H_{\mathrm{sw}}(-)$. Both $H_{\mathrm{sw}}(+)$ and $H_{\mathrm{sw}}(-)$ correspond to the $H_{\text {sw }}$ 's of the same NiFe layer, nevertheless there is great difference in magnitude between the $H_{\mathrm{sw}}(+)$ and the $H_{\mathrm{sw}}(-)$. Considering the difference of the relative magnetization configuration of the two layers, we can explain this difference as follows. Schematic illustrations of the magnetization configurations before and after the two $H_{\mathrm{sw}}$ 's are shown in Fig. 6. For simplicity, we assume that only the parallel component $H(\mathrm{Co})_{\|}$to the wire axis of the stray field from the magnetic charge of Co layer at the edge of the wire works on the $H_{\mathrm{sw}}$. We can then describe the $H_{\mathrm{sw}}(+)$ and $H_{\text {sw }}(-)$ as

$$
\left\{\begin{array}{c}
H_{\mathrm{sw}}(+)=H_{\mathrm{sw}}(0)-H(\mathrm{Co})_{\|} \\
\left|H_{\mathrm{sw}}(-)\right|=H_{\mathrm{sw}}(0)+H(\mathrm{Co}) \|
\end{array} \|\right.
$$

where $H_{\text {sw }}(0)$ is the $H_{\text {sw }}$ of the NiFe layer in the case of a single layer. Equation (2) explains that the magnitude of the $H_{\mathrm{sw}}(-)$ is larger than that of the $H_{\mathrm{sw}}(+)$ if the sign of $H(\mathrm{Co})_{\|}$is positive. Moreover from Eq. (2) we find that we can estimate

$$
H(\mathrm{Co})_{\|}=\frac{\left|H_{\mathrm{sw}}(-)\right|-H_{\mathrm{sw}}(+)}{2} .
$$

In Fig. 7(a) we plot the $H_{\mathrm{sw}}(+)$ 's and the $H_{\mathrm{sw}}(-)$ 's at $300 \mathrm{~K}$ as a function of the $\mathrm{Cu}$ layer thickness $X$ sandwiched between $\mathrm{NiFe}(20 \mathrm{~nm})$ layer and $\mathrm{Co}(20 \mathrm{~nm})$ layer. The $w$ of the measured wires in Fig. 7(a) is $150 \mathrm{~nm}$. In Fig. 7(b) we show the $H(\mathrm{Co})_{\|}$'s estimated from Eq. (3) using the experimental data in Fig. 7(a) and calculated $H(\mathrm{Co})_{\|}$'s based on the models I, II, and III (Fig. 8). For calculating the $H(\mathrm{Co}) \|$ 's we assumed three kinds of magnetization distribution in the $\mathrm{Co}(20 \mathrm{~nm})$ layer. Figures 8(a), 8(c), and 8(e) represent the top views of the assumed magnetization distribution at the left edge of the wire. Model I is the case of the homogeneous
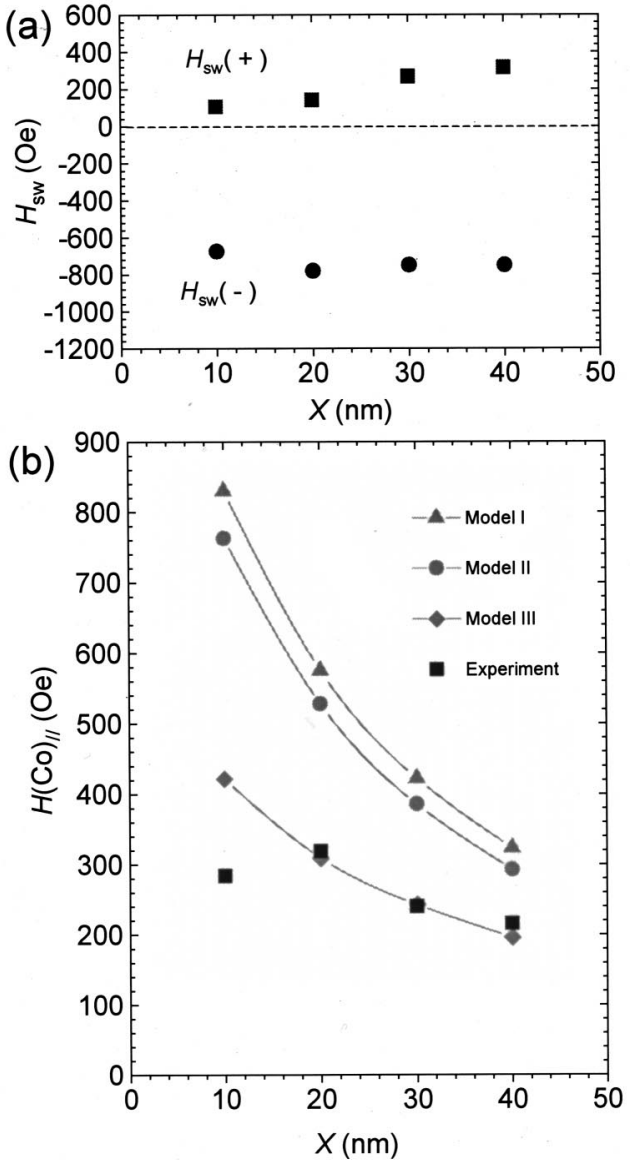

FIG. 7. (a) Cu layer thickness $X$ dependence of $H_{\mathrm{sw}}(-)$ and $H_{\mathrm{sw}}(+)$ at 300 $\mathrm{K}$. The measured wires $(w=150 \mathrm{~nm})$ consist of $\mathrm{NiFe}(20 \mathrm{~nm}) / \mathrm{Cu}(X \mathrm{~nm}) /$ $\mathrm{Co}(20 \mathrm{~nm})$. (b) $H(\mathrm{Co})_{\|}$as a function of $X$. Lines are guides to the eye.

distribution. The distributions in models II and III are typical ones reported in the previous reports ${ }^{15,16}$ and we also obtained them by solving the dynamic Landau-LifshitzGilbert equation to minimize exchange and magnetostatic energies. Figures $8(\mathrm{~b}), 8(\mathrm{~d})$, and $8(\mathrm{f})$ are the corresponding distributions of $H(\mathrm{Co})_{\|}$at the center of the NiFe layer for $X=20 \mathrm{~nm}$, assuming that the distance between two magnetic layers is $X+20 \mathrm{~nm}$. In the calculation of the $H(\mathrm{Co})_{\|}$we used a square mesh $4.7 \times 4.7 \mathrm{~nm}^{2}$. In Fig. $7($ b) we plotted the maximum values evaluated on the $\mathrm{NiFe}$ layer for $X$ $=10,20,30,40 \mathrm{~nm}$ and lines are guides to the eye. The calculated values by model III show nearly the same as the experimental values for $X=20,30,40 \mathrm{~nm}$, while the calculated values by models I and II show much larger values than the experimental values. This is due to the fact that magnetic charges in model III are less concentrated at the end of the wire than those in models I and II. From this result it is suggested that the magnetization distribution with vortex structure as we assumed in model III is realized in this experiment although the detail structure may be different. However, we are not sure of the reason why the experimental value for $X=10 \mathrm{~nm}$ is significantly smaller than the calculated value based on model III.

We also present the results of the dependence of $H_{\mathrm{sw}}$ (+) and $H_{\mathrm{sw}}(-)$ on $w$ for the wires consisting of $\mathrm{NiFe}(20$ $\mathrm{nm}) / \mathrm{Cu}(20 \mathrm{~nm}) / \mathrm{Co}(20 \mathrm{~nm})$ in Fig. 9(a). The dependence of 


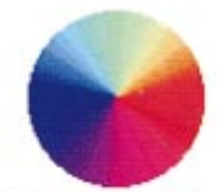

Magnetization Magnitude and direction direction

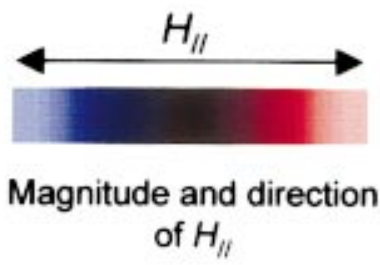

Model I

(a)

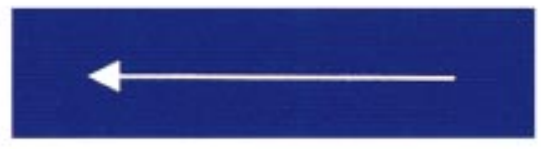

(b)

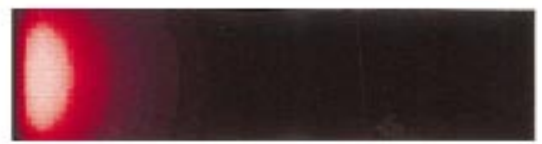

Model II

(c)

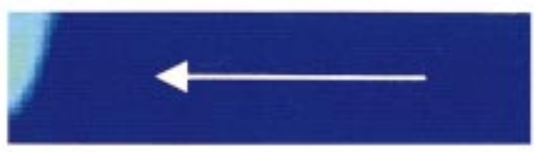

(d)

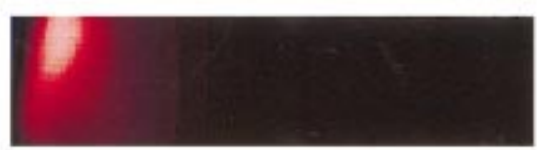

Model III

(e)

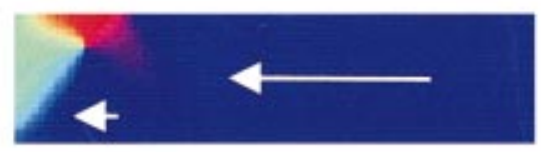

(f)

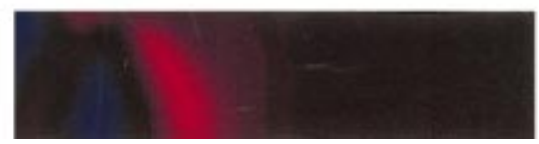

FIG. 8. (Color) (a), (c), (e) Top views of the assumed magnetization distribution of the Co(20 nm) layer at the left edge of the wire $(w=150 \mathrm{~nm})$. (b), (d), (f) Corresponding distributions of $H(\mathrm{Co})_{\|}$at the surface of the NiFe layer for $X=20 \mathrm{~nm}$.

the estimated $H(\mathrm{Co})_{\|}$from Eq. (3) using the experimental data in Fig. 9(a) on $w$ is shown in Fig. 9(b). The $H(\mathrm{Co})_{\|}$ decreases with increasing $w$. According to our calculation, the maximum value of $H_{\|}$does not depend on the width of the wire if we assume the similar distribution of magnetization. Probably our assumptions are too simple to understand our all results. For example, we consider only the parallel component of the stray field and moreover consider only the maximum value of the $H_{\|}$. Using the experimentally obtained $H(\mathrm{Co})_{\|}$values it is explained why the ratio of the two slopes in Fig. 4(a) is much larger than the expected value. Corrected $H_{\text {sw }}$ of the NiFe layer by considering the $H(\mathrm{Co})_{\|}$'s in Fig. 9(b) is also proportional to $1 / w$ and the ratio becomes the value of 1.7 which is very close to the expected value of 1.6 (Fig. 10).

The existence of the $H(\mathrm{Co})_{\|}$can also be the reason why attaching the square pad $\left(0.5 \times 0.5 \mu \mathrm{m}^{2}\right)$ at one end of the
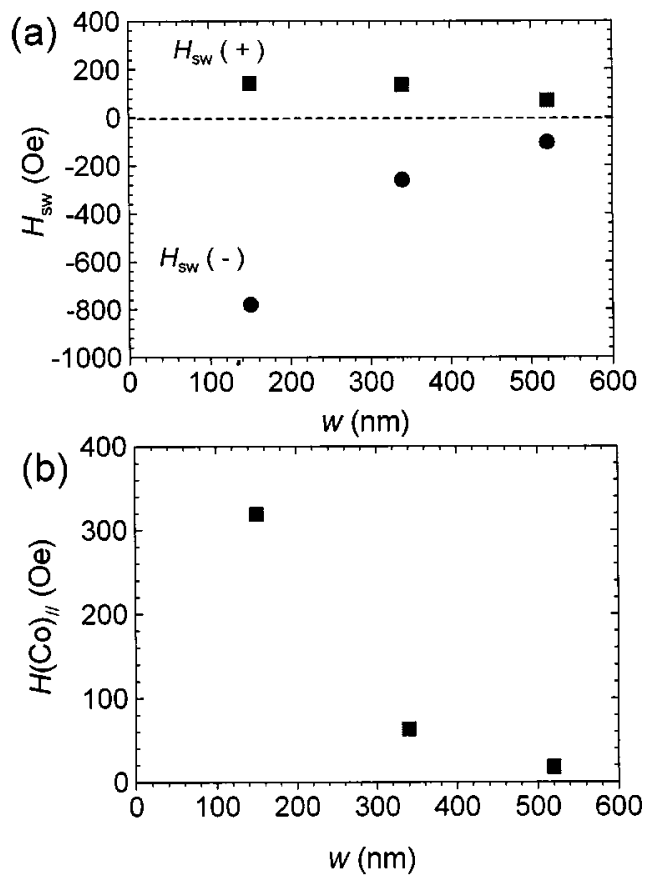

FIG. 9. (a) $H_{\mathrm{sw}}(-)$ and $H_{\mathrm{sw}}(+)$ as a function of wire width at $300 \mathrm{~K}$; (b) $H(\mathrm{Co})_{\|}$as a function of wire width. The measured wires consist of $\mathrm{NiFe}(20$ $\mathrm{nm}) / \mathrm{Cu}(20 \mathrm{~nm}) / \mathrm{Co}(20 \mathrm{~nm})$.

wire $(w=150 \mathrm{~nm})$ has no effect on the $H_{\mathrm{sw}}$ for the $\mathrm{NiFe}$ layer, while for the Co layer that results in the drastic decrease of the $H_{\mathrm{sw}}$ in Ref. 10. It is obvious that in the case of trilayer structure consisting of $\mathrm{NiFe} / \mathrm{Cu} / \mathrm{Co}$ magnetization of the NiFe layer switches under much lower applied field than in the case of a single layer structure by the assistance of the $H(\mathrm{Co})_{\|}$. The $H(\mathrm{Co})_{\|}$causes a larger decrease of $H_{\mathrm{sw}}$ than attaching a pad and therefore we cannot observe the effect of attaching a pad on the NiFe layer. Actually in the case of the $\mathrm{NiFe}$ wire with single layer structure, attaching a pad to the wire certainly has the effect of decreasing the switching field and the domain wall is always injected into the wire from the pad. $^{17,18}$

\section{Pad shape dependence of the wire with a pad}

For the wire $(w=150 \mathrm{~nm})$ consisting of $\mathrm{NiFe}(20 \mathrm{~nm}) /$ $\mathrm{Cu}(20 \mathrm{~nm}) / \mathrm{Co}(20 \mathrm{~nm})$ with a pad, we examined pad shape

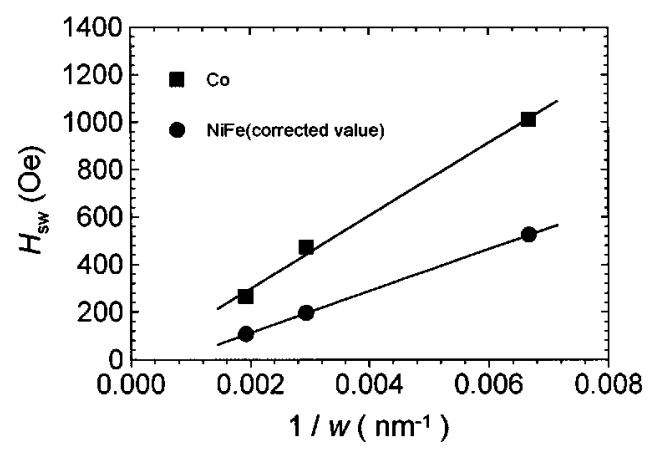

FIG. 10. Average $H_{\text {sw }}$ of the $\mathrm{NiFe}(20 \mathrm{~nm})$ layer and $\mathrm{Co}(20 \mathrm{~nm})$ layer as a function of the inverse of the wire width at $300 \mathrm{~K}$. The $H_{\mathrm{sw}}$ of the $\mathrm{NiFe}(20$ $\mathrm{nm})$ layer is the corrected value by considering the $H(\mathrm{Co})_{\|}$. 

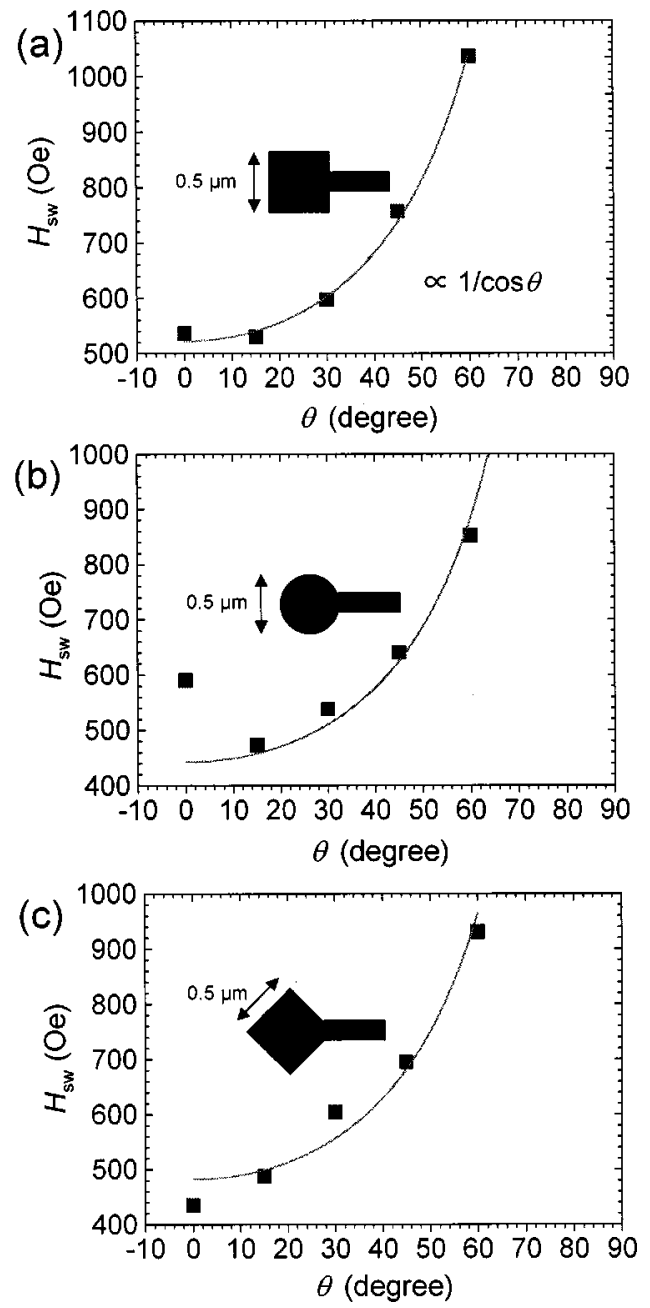

FIG. 11. Angular dependence of the $H_{\text {sw }}$ of the Co layer at $300 \mathrm{~K}$ for various wires with (a) a square pad; (b) a circle pad; and (c) a diamond pad. The pad shapes are schematically shown. The lines are fits to be proportional to $1 / \cos \theta$.

dependence of the $H_{\text {sw }}$ for the Co layer. The $H_{\text {sw }}$ for the Co layer at $300 \mathrm{~K}$ as a function of the angle $\theta$ between the wire axis and the applied field direction is shown in Fig. 11. The applied magnetic field is in the substrate plane. As schematically illustrated in Fig. 11, we prepared wires with three types of pad, (a) square pad, (b) circle pad, and (c) diamond pad. For all types of pad, the $H_{\mathrm{sw}}$ for the Co layer was much smaller than the $H_{\text {sw }}$ of about 1000 Oe in Fig. 2 for the wire without a pad. Therefore, we are sure that the domain wall nucleates in the pad at a smaller field than the nucleation field at the end of the wire and is pinned at the mouth of the wire until $H_{\text {sw }}$ at which field the wall is injected into the wire. ${ }^{10}$ In Fig. 12, a MFM image of the wire with a square pad is presented. This wire consists of a single Co(20 nm) layer. The image was obtained in absence of magnetic field. We are sure that a pinned wall exists at the mouth of the wire in Fig. 12. The purpose of specifying the direction of domain wall propagation in the magnetization reversal process ${ }^{10,17,18}$ is accomplished in all cases. However, for the angular dependence of the $H_{\text {sw }}$, the three types of wires show their respective features. The lines in Fig. 11 are fits based on the assumption that the $H_{\mathrm{sw}}$ is proportional to $1 / \cos \theta$. When the

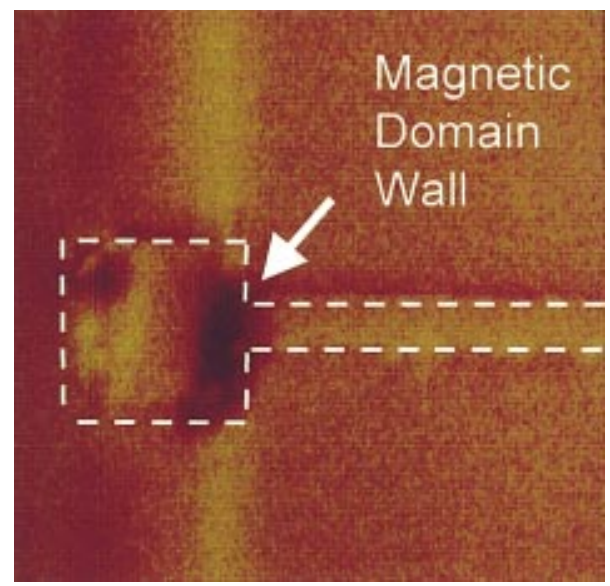

FIG. 12. (Color) MFM image for a wire with a square pad under zero field. This wire has a single layer structure of $\operatorname{Co}(20 \mathrm{~nm})$.

domain wall is pinned at the mouth of the wire, the effective field which pushes the pinned wall toward the wire axis is $\cos \theta$ of the applied field. While all data are on the line for the wire with a square pad, for the wire with a circle pad the $H_{\text {sw }}$ only for $\theta=0^{\circ}$ is much larger than the expected value and for the wire with a diamond pad the $H_{\text {sw }}$ 's for $\theta=0^{\circ}$ and $30^{\circ}$ are off the line. The data plotted in Fig. 11 are average values of the histograms shown in Fig. 13. The histograms obtained by repeating MR measurements 30 times give us more information. The distribution width for the wire with a square pad is generally narrower than that for the wire with a diamond pad. This result is probably attributed to how the wires are connected to the respective pads, because it is decided whether the mouth part of the wire works as a well defined pinning site for the magnetic domain wall or not over the entire range of the angle $\theta$. However, it seems that the reason of disagreement with the expected value at $\theta=0^{\circ}$ for the circle pad cannot be explained by this viewpoint because the histogram shows narrower distribution than that for the square pad. In this case there is a possibility that the tendency to be realized the flux-closure domain structure within the pad area plays an important role to decide the $H_{\mathrm{sw}}$. In the next section we report on the temperature dependence of the $H_{\mathrm{sw}}$ of the Co layer especially at $\theta=30^{\circ}$ for the wire with a square pad, for which we can describe the observed $H_{\text {sw }}$ by simply assuming that the effective field to the pinned wall is $\cos \theta$ of the applied field.

\section{Temperature dependence of the switching field for the wire with a square pad}

A wire $(w=150 \mathrm{~nm})$ with a square pad as shown in Fig. 1(b) was prepared. This wire is composed of $\operatorname{NiFe}(20 \mathrm{~nm}) /$ $\mathrm{Cu}(20 \mathrm{~nm}) / \mathrm{Co}(20 \mathrm{~nm})$. The sample was cooled down to $5 \mathrm{~K}$ under zero magnetic field and MR measurements at the angle $\theta=30^{\circ}$ were performed from 5 to $300 \mathrm{~K}$ with a constant current of $5 \mu \mathrm{A}$. The angle of $\theta=30^{\circ}$ was chosen because the histogram of the $H_{\mathrm{sw}}$ showed the narrowest distribution width for this angle. Prior to the MR measurement at a fixed temperature, a magnetic field of -3000 Oe was 

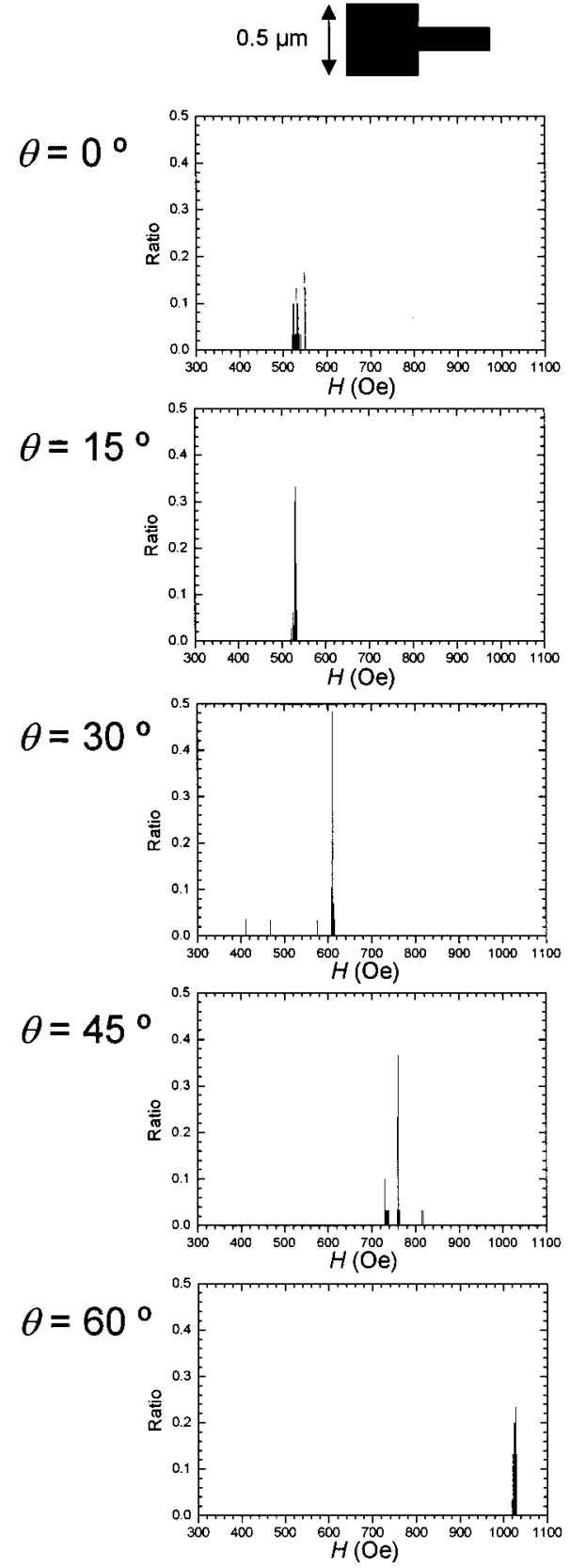
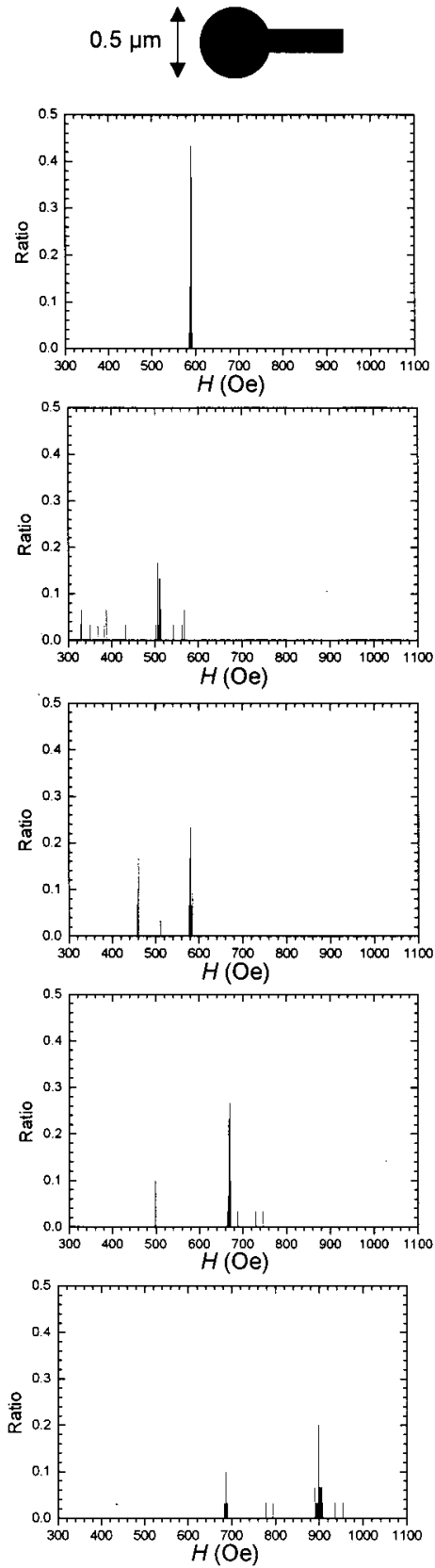
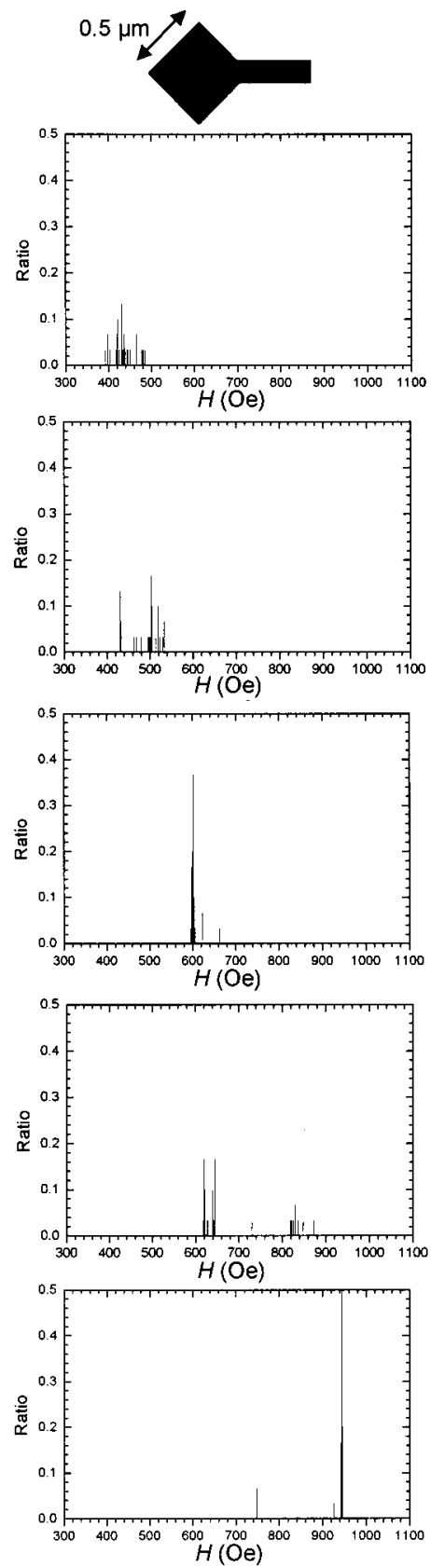

FIG. 13. Histograms of the $H_{\mathrm{sw}}$ 's of the Co layer at $300 \mathrm{~K}$. The data in Fig. 11 are the average values of the data in these histograms.

applied in order to align the magnetizations in one direction, negative direction (-). Then the resistance was measured with sweeping the field towards the counter direction, positive direction $(+)$, at the rate of $6 \mathrm{Oe} / \mathrm{s}$. The MR measurements were repeated 30 times to obtain the average $H_{\text {sw }}$ at each temperature. The inset of Fig. 14 shows the $H_{\text {sw }}$ 's of the Co layer as a function of temperature in two different measurement series, $(-)$ and $(+)$. The difference of the two measurement series is as follows. We distinguished the difference of the magnetization direction during cooling process down to 5 from $300 \mathrm{~K}$. There are two possible directions parallel to the wire axis. For the definition of two directions, we obeyed the definition of applied field direction. Namely in the series of $(+)$, in advance of the sample cooling down to $5 \mathrm{~K}$ under zero field, we aligned the magnetizations to the positive direction (+) by applying the field of 3000 Oe at $300 \mathrm{~K}$. On the other hand in the series of $(-)$, in advance of the cooling process we aligned the magnetizations to the negative direction (-) by applying the field of -3000 Oe at $300 \mathrm{~K}$. The direction of the magnetization is preserved when the applied field is reduced to zero. In the inset of Fig. 14 we notice that two series branch off at low temperatures below $50 \mathrm{~K}$. This difference of $H_{\mathrm{sw}}$ depending on the magnetization direction during cooling process can be caused by the oxidized (antiferromagnetic) surface layer. Unidirectional anisotropy for magnetic nanoparticles under field cooling have been reported by another groups. ${ }^{19-22}$ Figure 14 plots the average of two series, $[(-)+(+)] / 2$. By this procedure, we expect that the effect from the bias field caused by the oxidized surface layer is canceled. 


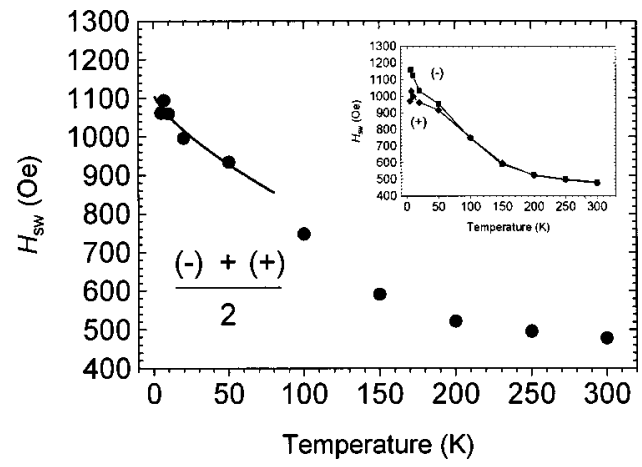

FIG. 14. Main figure: temperature dependence of the $H_{\mathrm{sw}}$ of the Co layer for the wire consisting of $\mathrm{NiFe}(20 \mathrm{~nm}) / \mathrm{Cu}(20 \mathrm{~nm}) / \mathrm{Co}(20 \mathrm{~nm})$ shown in Fig. 1(b). The average of $(-)$ and $(+)$ in the inset is plotted. The line is a fit using the Kurkijärvi model. Inset: temperature dependence of the $H_{\mathrm{sw}}$ in two different measurement series, $(-)$ and $(+) .(-)$ and $(+)$ correspond to the two different magnetization directions during cooling process. The lines are guides for the eye. The field is applied at an angle of $30^{\circ}$ with respect to the wire axis in the plane.

The line in Fig. 14 is a fit using the Kurkijärvi model ${ }^{23}$ which is based on the assumption of $\mathrm{Nee}^{24}$ on thermally assisted magnetization reversal over a simple potential barrier. This model fairly well describes our data at low temperatures below $50 \mathrm{~K}$. In this model the temperature dependence of the $H_{\text {sw }}$ is calculated to be $22,25-27$

$$
H_{\mathrm{sw}} \cong H_{0}\left\{1-\left[\frac{k T}{E_{0}} \ln \left(\frac{\Gamma_{0} k T H_{0}}{1.5 E_{0} \nu\left(1-H_{s w} / H_{0}\right)^{0.5}}\right)\right]^{2 / 3}\right\},
$$

where $\nu=6 \mathrm{Oe} / \mathrm{s}$ is the field sweep rate and $\Gamma_{0}$ is the attempt frequency. Values as $\Gamma_{0}$ in the range from $10^{8}$ to $10^{12}$ may be expected ${ }^{28}$ and we assume $\Gamma_{0}=10^{10} \mathrm{~Hz}$ in this article. In introducing Eq. (4), for the magnetic field dependence of energy barrier height, a simple analytical approximation is used: ${ }^{29}$

$$
E(H)=E_{0}\left(1-\frac{H}{H_{0}}\right)^{3 / 2},
$$

where $E_{0}$ is the energy barrier at zero field and $H_{0}$ is the switching field at zero temperature. From this fit using Eq. (4) with neglecting the $H_{\mathrm{sw}}$-dependent part in the denominator of the logarithmic term, we obtain $H_{0}=1117 \mathrm{Oe}$ and $E_{0}$ $=16000 \mathrm{~K}$. On the other hand the data at higher than $50 \mathrm{~K}$ cannot be fit by Eq. (4). The reason is not clear at the present stage. However, in the model used here, it is assumed that the magnetization reversal proceeds only through the lowest energy pass. Therefore, there is a possibility that in higher region of temperatures the assumption is not suitable.

Finally we point out a marked result on the $H_{\mathrm{sw}}$ distribution dependence on temperature. As shown in Fig. 15 the distribution width of the $H_{\mathrm{sw}}$ 's drastically changes between 100 and $150 \mathrm{~K}$. The histograms for both the (-) series and $(+)$ series show the same tendency. Our sample has a polycrystalline structure with grain sizes of about $10 \mathrm{~nm}$ and the width of the domain wall in our sample is expected to be the same size as the wire width. ${ }^{30,31}$ This means that there are more than 200 grains in the area of the pinned magnetic domain wall. Because magnetocrystalline anisotropy exists in each grain, it seems that the magnetic domain wall pinned
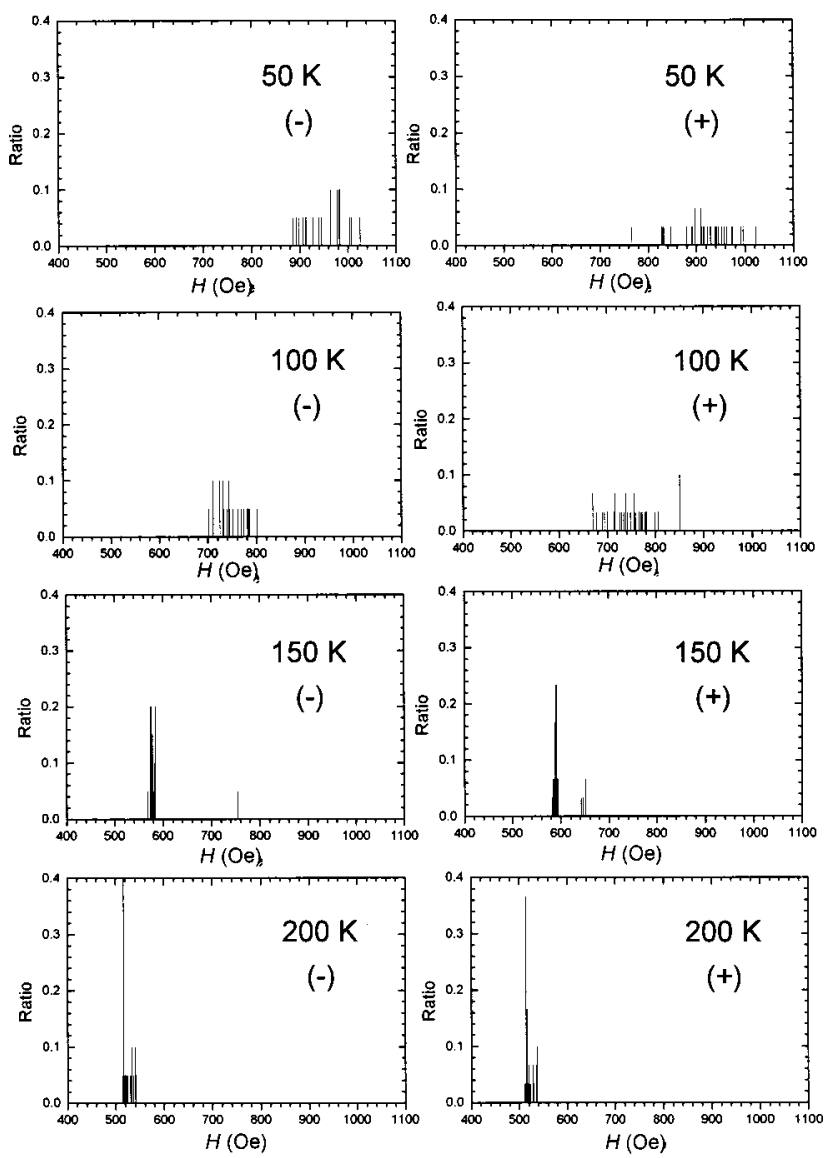

FIG. 15. Histograms of the $H_{\mathrm{sw}}$ 's of the Co layer at each temperature. The data in Fig. 14 are the average values of the data in these histograms.

between the pad and the wire has different structure in detail at every field sweep and this causes the drastic broadening of the distribution width below $100 \mathrm{~K}$. Fortunately this value of $100 \mathrm{~K}$ is much smaller than $16000 \mathrm{~K}$ estimated as $E_{0}$. This is probably the reason why the model assuming only a single potential barrier describes well the temperature dependence of the average $H_{\mathrm{sw}}$.

\section{CONCLUSION}

We measured the magnetization switching fields of a single magnetic wire consisting of $\mathrm{NiFe} / \mathrm{Cu} / \mathrm{Co}$ using noncoupled type GMR effect. For both magnetic layers, we observed that the switching field is inversely proportional to the wire width $(150-520 \mathrm{~nm})$. The measured switching field had the character of a stochastic variable and the distribution width decreases with decreasing the width of the wire.

We obtained the much different values as the switching field of the same $\mathrm{NiFe}$ layer corresponding to the relative magnetization configurations between $\mathrm{NiFe}$ and Co layers. This can be explained by the stray field from the magnetic charge of the Co layer at the edge of the wire. This effect is decreased with increase of the sandwiched $\mathrm{Cu}$ layer thickness. Moreover, this effect is decreased with an increase of the wire width according to the fact that magnetic charges distribute over a wider area around the edge of the wire with increase of the wire width. 
For all types of pads investigated in this study, attaching a pad at one end of the wire causes a drastic decrease of the switching field for the Co layer at $300 \mathrm{~K}$, though attaching a pad had no effect on the NiFe layer. In magnetization switching of the Co layer a magnetic domain wall is always injected from the pad into the wire. The wire with the square pad shows the most reasonable angular dependence of the switching field on the assumption that the switching field corresponds to the depinning field of the pinned wall from the mouth of the wire. For this wire we measured the temperature dependence of the switching field between 5 and $300 \mathrm{~K}$. The dependence at low temperatures between 5 and $50 \mathrm{~K}$ can be described by the model on thermally assisted magnetization reversal over a simple potential barrier. The distribution width of the switching fields drastically changed between 100 and $150 \mathrm{~K}$. This is probably attributed to the fact that our sample has a polycrystalline structure.

\section{ACKNOWLEDGMENTS}

The authors would like to thank Professor H. Miyajima, Professor N. Hosoito, and Professor K. Mibu for valuable discussions. The present work was supported by Grants-inAid for Scientific Research on Priority Area and Creative Basic Research from Monbusho and by the NEDO Project. One of the authors (K.S.) was supported by the JSPS Research Fellowship For Young Scientists.

${ }^{1}$ M. Lederman, S. Shultz, and M. Ozaki, Phys. Rev. Lett. 73, 1986 (1994).

${ }^{2}$ C. Salling, R. O'Barr, S. Schultz, I. McFadyen, and M. Ozaki, J. Appl. Phys. 75, 7989 (1994).

${ }^{3}$ T. Hirayama, Q. Ru, T. Tanji, and A. Tonomura, Appl. Phys. Lett. 63, 418 (1993).

${ }^{4}$ A. K. Geim, S. V. Dubonos, J. G. S. Lok, I. V. Grigorieva, L. Theil Hansen, and P. E. Lindelof, Appl. Phys. Lett. 71, 2379 (1997)

${ }^{5}$ W. Wernsdorfer, K. Hasselbach, A. Benoit, B. Barbara, D. Mailly, J. Tuaillon, J. P. Perez, V. Dupuis, J. P. Dupin, G. Guiraud, and A. Perez, J. Appl. Phys. 78, 7192 (1995).

${ }^{6}$ W. Wernsdorfer, B. Doudin, D. Mailly, K. Hasselbach, A. Benoit, J.
Meier, J. P. Ansermet, and B. Barbara, Phys. Rev. Lett. 77, 1873 (1996).

${ }^{7}$ T. Ono, H. Miyajima, K. Shigeto, and T. Shinjo, Appl. Phys. Lett. 72, 1116 (1998).

${ }^{8}$ See, e.g., M. E. Schabes, J. Magn. Magn. Mater. 95, 249 (1991), and references therein.

${ }^{9}$ T. Ono, H. Miyajima, K. Shigeto, K. Mibu, N. Hosoito, and T. Shinjo, Science 284, 468 (1999).

${ }^{10}$ K. Shigeto, T. Shinjo, and T. Ono, Appl. Phys. Lett. 75, 2815 (1999).

${ }^{11}$ J. Osborn, Phys. Rev. 67, 351 (1945).

${ }^{12}$ A. Maeda, M. Kume, T. Ogura, K. Kuroki, T. Yamada, M. Nishikawa, and Y. Harada, J. Appl. Phys. 76, 6667 (1994).

${ }^{13}$ A. O. Adeyeye, J. A. C. Bland, C. Daboo, J. Lee, U. Ebels, and H. Ahmed, J. Appl. Phys. 79, 6120 (1996).

${ }^{14}$ E. Y. Chen, S. Tehrani, T. Zhu, M. Durlam, and H. Goronkin, J. Appl. Phys. 81, 3992 (1997).

${ }^{15}$ K. J. Kirk, J. N. Chapman, and C. D. W. Wilkinson, Appl. Phys. Lett. 71, 539 (1997).

${ }^{16}$ J. Shi, S. Tehrani, T. Zhu, Y. F. Zheng, and J.-G. Zhu, Appl. Phys. Lett. 74, 2525 (1999).

${ }^{17}$ Y. Yokoyama, Y. Suzuki, S. Yuasa, K. Ando, K. Shigeto, T. Shinjo, P. Gogol, J. Miltat, A. Thiaville, T. Ono, and T. Kawagoe, J. Appl. Phys. 87, 5618 (2000)

${ }^{18}$ W. Y. Lee, C. C. Yao, A. Hirohata, Y. B. Xu, H. T. Leung, S. M. Gardiner, S. McPhail, B. C. Choi, D. G. Hasko, and J. A. C. Bland, J. Appl. Phys. 87, 3032 (2000).

${ }^{19}$ W. H. Meiklejohn and C. P. Bean, Phys. Rev. 105, 904 (1957).

${ }^{20}$ R. H. Kodama, A. E. Berkowitz, E. J. McNiff, Jr., and S. Foner, Phys. Rev. Lett. 77, 394 (1996)

${ }^{21}$ J. G. S. Lok, A. K. Geim, J. C. Maan, L. T. Kuhn, and P. E. Lindelof, Phys. Rev. B 58, 12201 (1998).

${ }^{22}$ J. G. S. Lok, A. K. Geim, U. Wyder, J. C. Maan, and S. V. Dubonos, J. Magn. Magn. Mater. 204, 159 (1999).

${ }^{23}$ J. Kurkijärvi, Phys. Rev. B 6, 832 (1972).

${ }^{24}$ L. Néel, Ann. Geophys. (C.N.R.S.) 5, 99 (1949).

${ }^{25}$ L. Gunther and B. Barbara, Phys. Rev. B 49, 3926 (1994).

${ }^{26}$ A. Garg, Phys. Rev. B 51, 15592 (1995).

${ }^{27}$ W. Wernsdorfer, K. Hasselbach, A. Benoit, B. Barbara, B. Doudin, J. Meier, J.-Ph. Ansermet, and D. Mailly, Phys. Rev. B 55, 11552 (1997).

${ }^{28}$ G. Bertotti, Hysteresis in Magnetism (Academic, New York, 1998), p. 333.

${ }^{29}$ R. H. Victora, Phys. Rev. Lett. 63, 457 (1989).

${ }^{30}$ R. D. McMichael and M. J. Donahue, IEEE Trans. Magn. 33, 4167 (1997)

${ }^{31}$ Y. Nozaki, K. Matsuyama, T. Ono, and H. Miyajima, Jpn. J. Appl. Phys., Part 1 38, 6282 (1999). 